Renana Wilkof Segev, Milana Gelman, Esther Maor-Sagie, Alon Shrim, Mordechai Hallak and Rinat Gabbay-Benziv*

\title{
New reference values for biometrical measurements and sonographic estimated fetal weight in twin gestations and comparison to previous normograms
}

https://doi.org/10.1515/jpm-2019-0207

Received June 22, 2019; accepted July 15, 2019; previously published online August 2, 2019

\section{Abstract}

Objective: To construct new reference values for biometrical measurements and sonographic estimated fetal weight (sEFW) in twin gestations and compare them to previously published normograms.

Methods: A retrospective analysis of sEFW evaluations of twin gestations was performed between 2011 and 2016 in a single university-affiliated medical center. sEFW was calculated using the Hadlock 1985 formula. To avoid selection bias, one evaluation per pregnancy was randomly selected. Following mathematical transformation to obtain normality of values, normograms were constructed using a best-fit regression model for estimation of mean and standard deviation at each gestational age (GA). Normograms were validated by applying all observations to ensure equal distribution at parallel percentiles. Our normograms were then compared to previously published sEFW normograms for twin gestations.

Results: A total of 864 sEFW evaluations were performed on 195 twin pregnancies at 22-39 gestational weeks. Of them, 390 entered the primary analysis. The rest were left for validation. Seventy percent of the cohort were dichorionic-diamniotic twins (136/195), 16\% (32/195) were monochorionic-diamniotic twins and three (1.5\%) were monochorionic-monoamniotic twins. Twenty-four fetuses lacked data on chorionicity. The rest were monochorionic

\footnotetext{
*Corresponding author: Rinat Gabbay-Benziv, MD, Department of Obstetrics and Gynecology, Hillel Yaffe Medical Center, Hadera 38100, Israel; and The Ruth and Bruce Rappaport Faculty of Medicine, Technion Israel Institute of Technology, Haifa, Israel, Tel.: +972-4-6304304/Mobile: +972-52-2222778, E-mail: gabbayrinat@gmail.com. https://orcid.org/0000-00016887-6314

Renana Wilkof Segev, Milana Gelman, Esther Maor-Sagie, Alon Shrim and Mordechai Hallak: Department of Obstetrics and Gynecology, Hillel Yaffe Medical Center, Hadera 38100, Israel; and The Ruth and Bruce Rappaport Faculty of Medicine, Technion Israel Institute of Technology, Haifa, Israel. https://orcid.org/0000-00021726-3830 (R. Wilkof Segev)
}

twins or were of unknown chorionicity. Values corresponding to the $2.5^{\text {th }}, 10^{\text {th }}, 50^{\text {th }}, 90^{\text {th }}$ and $97.5^{\text {th }}$ percentiles for sEFW are presented for every GA. Validation by applying all 864 evaluations on constructed normograms was achieved. Comparison to previously published twins' sEFW normograms demonstrated wide variation between curves.

Conclusion: New reference values for biometrical measurements and sEFW in twin gestations are presented for clinical and research use. Comparison to other curves demonstrates the wide variability and need for further investigation on twin's normal growth.

Keywords: biometrical measurements; birth weight normograms; estimated fetal weight; reference values; twin gestation.

\section{Introduction}

Twin gestations comprise 2-3\% of all deliveries; however, their contribution to neonatal morbidity and mortality is disproportionally large [1-4]. Fetal growth abnormalities, which are more common among twins, as compared to singleton gestations [5] are one of the top reasons for preterm delivery and adverse perinatal outcome. Meticulous surveillance is therefore needed, and most guidelines suggest continuous fetal growth surveillance by at least monthly ultrasound scan for all twin pregnancies [6].

The diagnosis of deviation from the normal growth pattern, whether in singleton or twin gestations, lies on reference values, that are mathematically constructed based on different combinations of fetal biometrical measurements multiplied by their calculated coefficients. Fetal biometrical measurements mainly include the head circumference (HC), biparietal diameter (BPD), abdominal circumference (AC) and the femur length (FL). Previous studies that evaluated reference values for estimation of fetal weight in twin compared to singleton fetuses demonstrated different growth pattern for twins, especially at the third trimester with lower accuracy in predicting the actual birth weight $[7,8]$. Hence, it is recommended to use twins-specific growth reference values. 
Data on biometric measurements in twin gestations are scarce. Moreover, most of the previously published reference values for estimated fetal weight in twin gestations are population-based, generated from birth weights. These share the disadvantage of underestimating fetal growth restriction as birth weights, especially among preterm infants, often represent a subset population in which a pathological process led to preterm delivery [912]. Only a few ultrasound-based twin growth reference values exist $[9,10,13-16]$, all were constructed in different clinical settings using different statistical methods. Thus, in this study, we sought to construct new reference values for biometrical measurements and sonographic estimation of fetal weight (sEFW) in twin gestations and compare them to previously published normograms.

\section{Materials and methods}

We conducted a retrospective cross-sectional analysis of SEFW in twin gestations at a single university-affiliated medical center between January $1^{\text {st }}, 2011$ and December $31^{\text {th }}$ 2016. All sEFW evaluations for both fetuses throughout pregnancy were manually retrieved and verified by study personnel. Inclusion criterion were twin pregnancies evaluated between $22+0$ and $39+6$ weeks of gestation with gestational age (GA) calculated by the last menstrual period or by first trimester ultrasound if discrepancy between them exceeded 7 days. We excluded cases that underwent early fetal reduction or were diagnosed with major congenital fetal anomalies or chromosomal abnormalities. No exclusions were made on grounds of chorionicity, growth abnormalities, maternal ethnicity, hypertension or diabetes. The study was approved by the Institutional Review Board. Due to the retrospective nature of the study, consent form was not necessary.

By convention, our ambulatory maternal fetal medicine clinics follow twin gestations from first to last trimester, depending on the time of first referral. Follow-up includes a once-a-month visit for dichorionic twins and once-in-two weeks visit for monochorionic twins until delivery. Every visit includes an ultrasound evaluation for fetal growth as well as a physician consultation. Uncomplicated low-risk twin gestations are offered the same follow-up. All ultrasound examinations are performed at the ultrasound unit by senior physicians who are ultrasound specialists or by experienced ultrasound technicians. Examinations are performed using General Electric Voluson E6 or E8 (Milwaukee, WI, USA) machines in a standardized fashion, according to the American Institute of Ultrasound in Medicine (AIUM) and the International Society of Ultrasound in Obstetrics and Gynecology (ISUOG) guidelines with measurements of the FL, BPD, HC and AC [11, 12, 17]. sEFW is calculated using the Hadlock et al. formula from 1985 [18]:

For every sEFW evaluation, GA was calculated and presented in decimals, rounded to the nearest tenth. To avoid selection bias, one ultrasound examination (with one weight estimation for each twin) was selected randomly per patient. Once a patient was assigned to a GA, her other examinations were excluded. For calculation of the regression of the mean and standard deviation (SD), reference values were constructed on the basis of the method described by Altman and Chitty [19] and Royston and Wright [20]. Data were not normally distributed and required transformation to achieve a normal distribution. This was accomplished by performing a power transformation $\left[x(\lambda)=x^{\lambda}\right]$ [21] on each estimated weight. After creating the regression for the mean and SD, values were back-transformed to natural numbers using the opposite mathematical operation. The best-fit regression model for the mean was derived from a polynomial regression fit for GA [19]. The best-fit model was decided according to the lowest residual SD. Analysis using fractional polynomials was applied but it did not improve the fit. For calculation of the SD, first scale absolute residuals were calculated for each measurement [(absolute value of measurement - estimated value of mean) $\times 1.2]$ [19]. The SD for each GA was obtained by a linear regression of the residuals by GA.

The model fit was then assessed by validating the normal distribution of the $\mathrm{Z}$ score, which was obtained from the mean and SD of the models. The $2.5^{\text {th }}, 10^{\text {th }}, 90^{\text {th }}$ and $97.5^{\text {th }}$ percentiles at each gestational week were then derived by the relationship mean $\pm Z^{\star} \times S D$, where $Z^{\star}$ is the standard normal deviation with \pm 1.282 for the $10^{\text {th }}$ and $90^{\text {th }}$ percentiles, and \pm 1.96 for the $2.5^{\text {th }}$ and $97.5^{\text {th }}$ percentiles. To avoid the inclusion of biologically implausible data points in the analysis, outliers that were $>3.5 \mathrm{SD}$ from the mean at each week were excluded. All calculations were performed with power-transformed values. The obtained values were then back-transformed and used for the construction of reference values.

To validate the fit of our references, we applied all growth estimations available (including all results available for every pregnancy) to the constructed reference values to ensure equal distribution at parallel percentiles (for example, the $10^{\text {th }}$ and $90^{\text {th }}$ percentiles).

For the biometrical measurements, AC, FL and HC, normograms were constructed using the same methodology described earlier. For clinical practice, normograms for FL and AC were presented as percentiles, and for the $\mathrm{HC}$ as -1 and -2 SD from the means.

New sEFW normograms were plotted and compared with six previously published twin gestation growth normograms. Three of them were constructed from prospective cohorts - Grantz et al. (2012-2013, USA, $n=171$ ) [9], Yarkoni et al. (1987, USA, $n=35)$ [13] and Liao et al. (2007-2010, Brazil, $\mathrm{n}=125)$ [14] and three from a retrospective design - Araujo et al. (2006-2013, Brazil, $\mathrm{n}=176)$ [10], Shivkumar et al. (1996-2006, Canada, $\mathrm{n}=321$ ) [15] and Gabbay-Benziv et al. (2006-2012, USA, $n=1427$ ) [16]. All normograms, except for Araujo et al., used a longitudinal analysis and all studies, except for Yarkoni et al., included dichorionic-diamniotic twins only. To facilitate comparison, charts are expressed graphically. Also, we present normograms as the SD scores based on Grantz et al.'s [9] study, the largest prospective data published on US population [the Eunice Kennedy Shriver National Institute of Child Health and Human Development (NICHD) fetal growth study].

Fetal biometrical measurements were similarly compared to Chervenak et al.'s curves [22] for HC and Hadlock et al.’s curves [18] for AC.

Data analysis was performed with Microsoft Excel (Microsoft Corporation, Redmond, WA, USA) and SPSS version 21.0 software (IBM Corporation, Chicago, IL, USA).

\section{Results}

A total of $864 \mathrm{sEFW}$ evaluations from 195 twin pregnancies between $22+0$ and $39+6$ weeks of gestation were evaluated. After exclusion of repeat examinations per patient, 
390 sEFW assessments were used for the final analysis. Fifty-six percent of the cohort (110 twin gestations) were from the Jewish population and 44\% (85 twin gestations) were from the Arab population. The median maternal age was 28 years and median GA at sonographic weight evaluation was 30.57 weeks of gestation (range 22.14-39.14). From the overall 195 twin gestations, 136 (69.7\%) were dichorionic-diamniotic gestations, 32 (16.4\%) were monochorionic-diamniotic twins and three (1.5\%) were monochorionic-monoamniotic twins. Twenty-four fetuses lacked chorionicity data in our computerized database. One pregnancy was complicated by stage 1 twin to twin transfusion syndrome that resolved spontaneously.

Fetal weight estimations were not normally distributed (Shapiro-Wilk $\mathrm{W}$ test, $\mathrm{P}<0.0001$; skewness $=0.34$ ); therefore, data were normalized using the square root transformation (skewness $=0.01$ ). A linear regression was used for the construction of sEFW normograms (equations: $=2.018 \times \mathrm{GA}-22.59$ for $\mathrm{sEFW}, \mathrm{R}^{2}=0.931$; and $=0.119 \times \mathrm{GA}-$ 1.402 for the SD). Using the aforementioned calculations, the $50^{\text {th }}, 2.5^{\text {th }}, 10^{\text {th }}, 90^{\text {th }}$ and $97.5^{\text {th }}$ percentiles were calculated for each GA and are presented in Figure 1 and Table 1. Using these references, sEFW can be classified as normal if within the $10^{\text {th }}-90^{\text {th }}$ percentiles for GA (by the table available), or score percentile can be calculated individually for every measurement using a Z-score: To evaluate the goodness of fit for our reference values, we applied all 864 fetal weight estimations (average 4.4 examinations per patient) on the constructed normograms (Figure 2).

We then compared our references values to six previously published sEFW curves $[9,10,13-15]$ of twin gestations (Figures 3 and 4). Most curves yielded similar sEFW until about 32-33 weeks of gestation when curves by Gabbay-Benziv et al. [16], Liao et al. [14] and Yarkoni et al. [13] deviated toward lower sEFW values for every GA. Shivkumar et al.'s [15] curve demonstrated higher sEFW values at all GA compared to all other curves. Reference values of the present study were similar to those of Araujo et al. [10] and Gabbay-Benziv et al. [16], with smallest deviation from Grantz et al.'s [9] normograms; repeat analysis for the AC, FL and HC yielded the reference values, which are presented in Figure 1B-D and Table 2. Comparisons to currently in use international reference values are presented in Figure 5.

\section{Discussion}

In this study, we sought to construct a new set of reference values for fetal biometrical measurements and sEFW for unselected twin gestations and compare them to previously published normograms. Our results demonstrate fetal growth pattern with an almost linear increase in
A

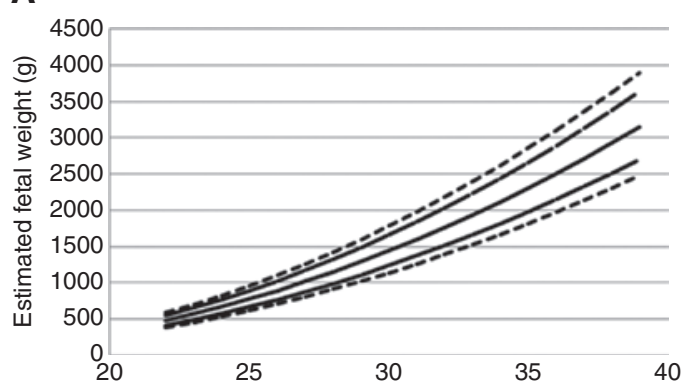

C

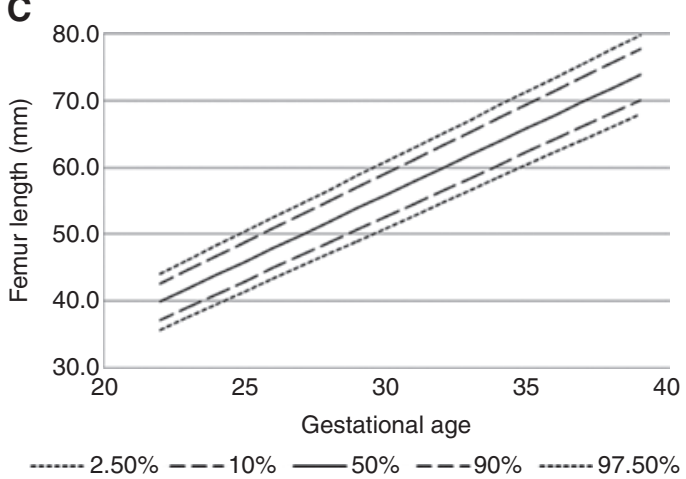

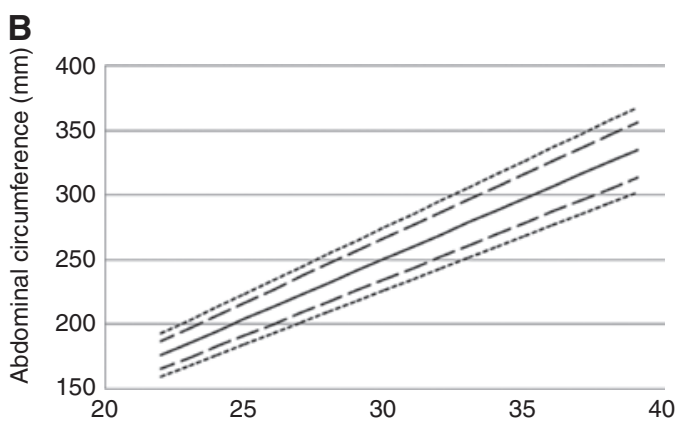

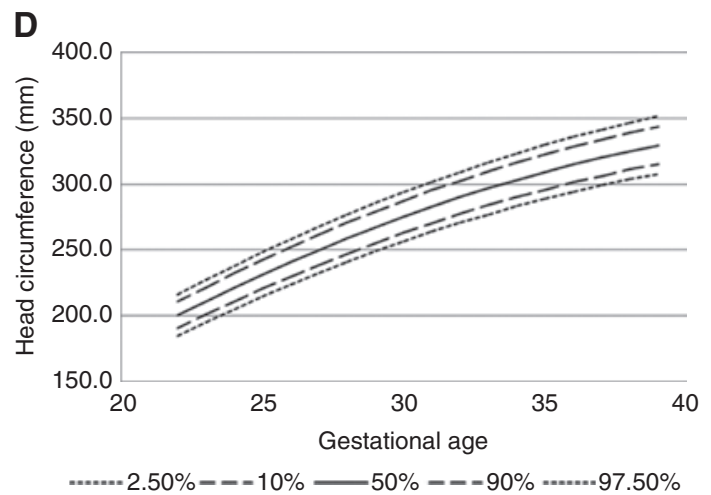

Figure 1: New set of reference values for sonographic estimated fetal weight and main biometrical measurements in twin gestation. (A) Sonographic estimated fetal weight; (B) abdominal circumference; (C) femur length; (D) head circumference. 
Table 1: New set of reference values for sonographic estimated fetal weight in twin gestations.

\begin{tabular}{|c|c|c|c|c|c|c|}
\hline Gestational week & $\mathrm{n}$ & $2.5^{\text {th }}$ centile, $\mathrm{g}$ & $10^{\text {th }}$ centile, $\mathrm{g}$ & $50^{\text {th }}$ centile, $\mathrm{g}$ & $90^{\text {th }}$ centile, $\mathrm{g}$ & $97.5^{\text {th }}$ centile, $\mathrm{g}$ \\
\hline 22 & 13 & 377 & 410 & 476 & 546 & 585 \\
\hline 23 & 7 & 450 & 489 & 568 & 652 & 699 \\
\hline 24 & 11 & 529 & 575 & 668 & 768 & 823 \\
\hline 25 & 11 & 614 & 668 & 776 & 893 & 957 \\
\hline 26 & 12 & 706 & 768 & 893 & 1027 & 1102 \\
\hline 27 & 9 & 804 & 875 & 1017 & 1171 & 1256 \\
\hline 28 & 12 & 908 & 988 & 1150 & 1324 & 1421 \\
\hline 29 & 14 & 1019 & 1109 & 1291 & 1487 & 1596 \\
\hline 30 & 10 & 1136 & 1237 & 1440 & 1659 & 1781 \\
\hline 31 & 12 & 1259 & 1372 & 1597 & 1840 & 1976 \\
\hline 32 & 12 & 1389 & 1513 & 1763 & 2031 & 2181 \\
\hline 33 & 14 & 1525 & 1662 & 1936 & 2232 & 2396 \\
\hline 34 & 13 & 1668 & 1818 & 2118 & 2442 & 2622 \\
\hline 35 & 16 & 1817 & 1980 & 2308 & 2661 & 2857 \\
\hline 36 & 11 & 1972 & 2150 & 2506 & 2889 & 3103 \\
\hline 37 & 12 & 2134 & 2326 & 2712 & 3127 & 3359 \\
\hline 38 & 4 & 2302 & 2509 & 2926 & 3375 & 3625 \\
\hline 39 & 2 & 2476 & 2700 & 3149 & 3632 & 3901 \\
\hline
\end{tabular}

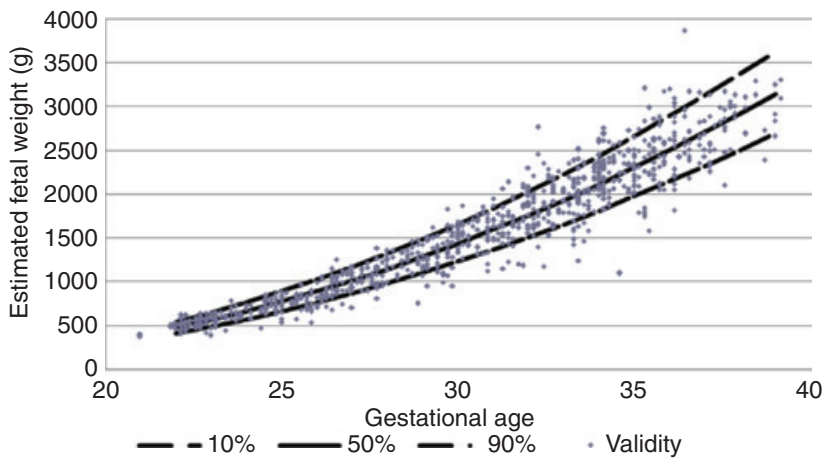

Figure 2: Validation of estimated fetal weight reference values by all 864 examinations.

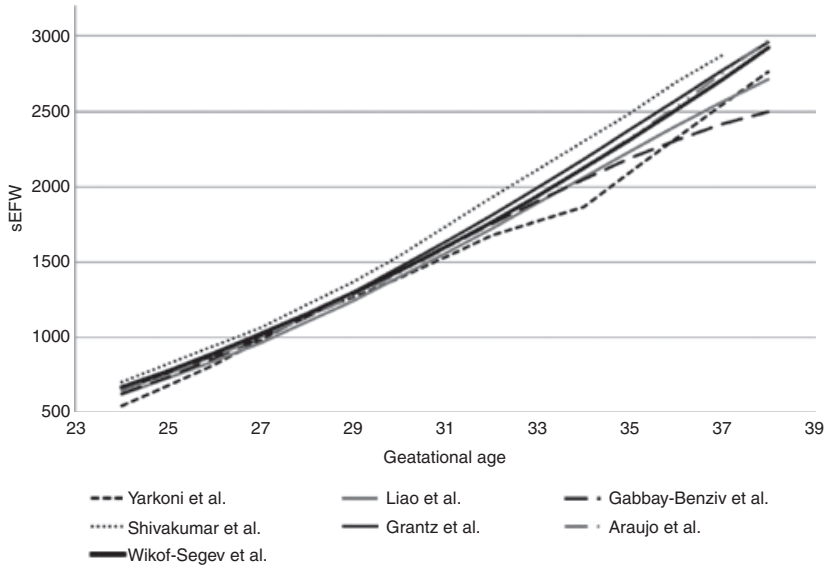

Figure 3: Comparison of various sonographic estimated fetal weight normograms in twin gestations. fetal weight throughout pregnancy. Values corresponding to major percentiles are presented for every GA (Table 1). Appropriateness of reference values is presented applying 864 examinations on constructed normograms. Comparison of sEFW to previously published curves demonstrated considerable variation across curves.

Only a few previous studies published sEFW reference values for twin growth. These represented different cohorts in terms of nationality, ethnicity, chorionicity and also differed by their methodology. Therefore, it is not surprising to have some variation between them. However, the magnitude of variation may raise questions about their validity and generalizability.

The first twins' sEFW normograms for US population were published in the 1990s by Yarkoni et al. [13]. Only 35

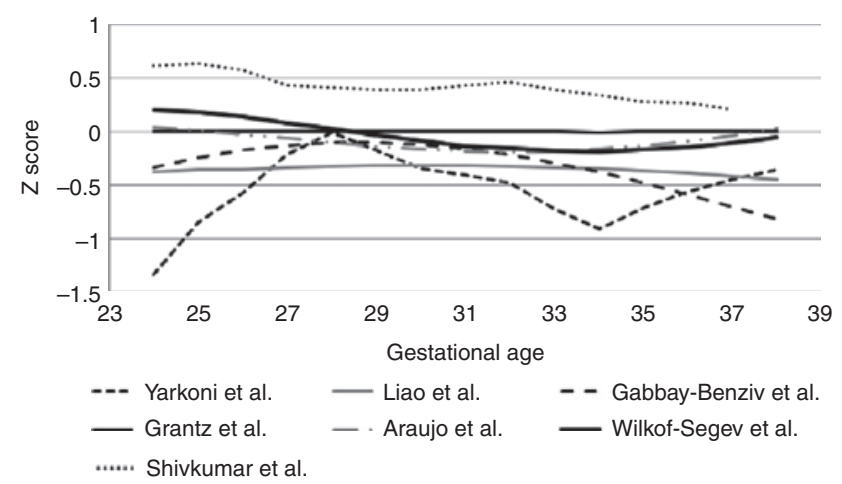

Figure 4: Comparison of reference values expressed as Grantz et al.'s [9] SD scores.

SD, standard deviation. 


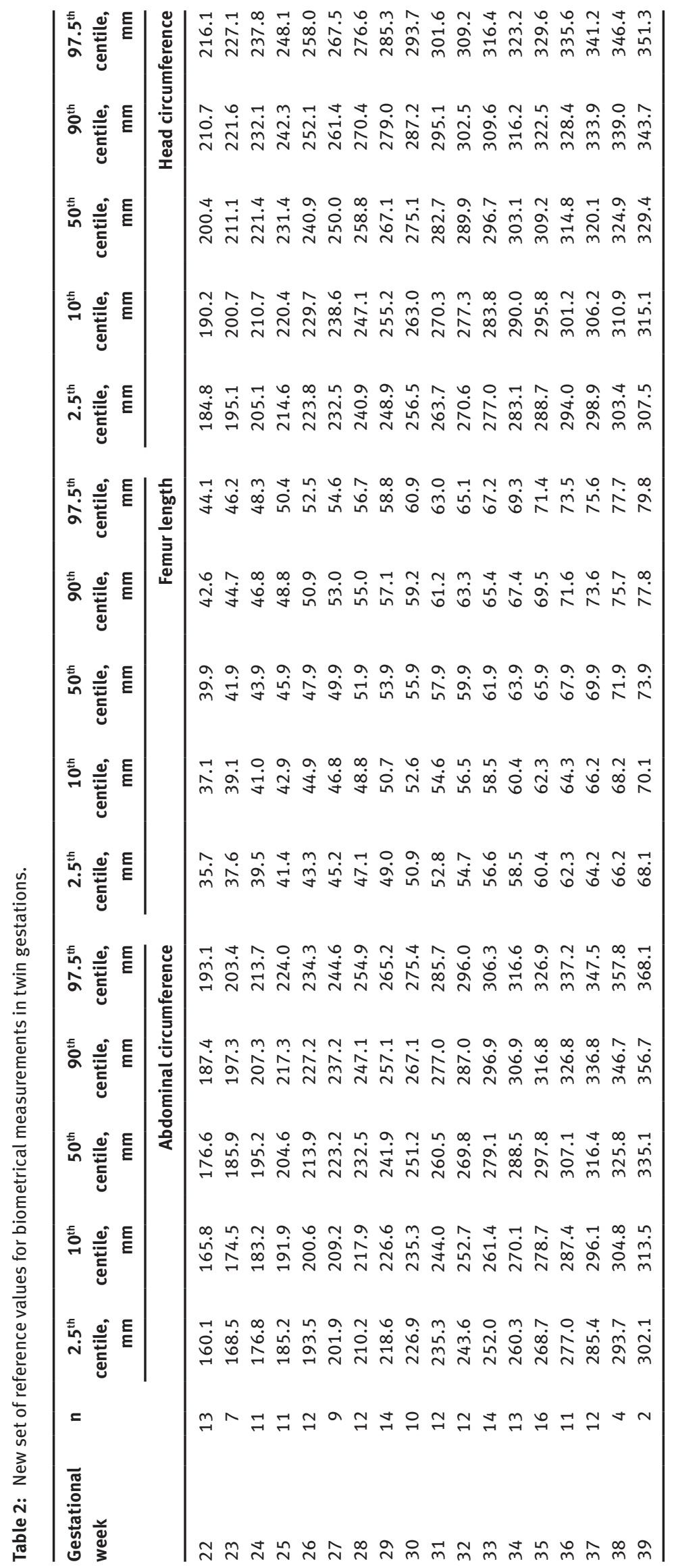



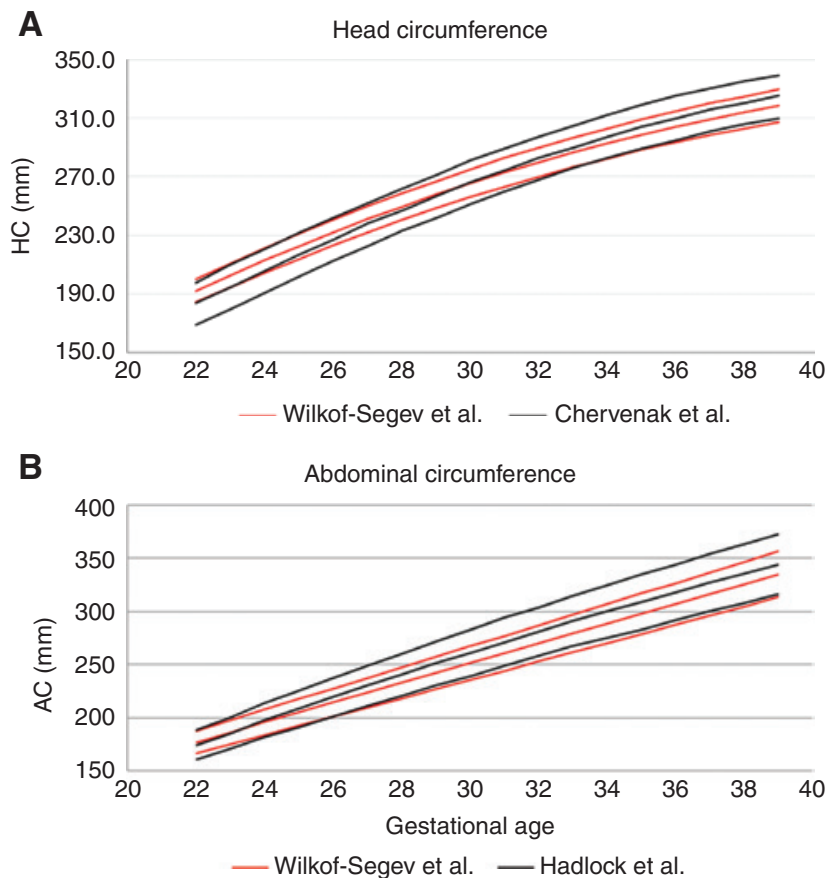

Figure 5: Comparison of reference values of sonographic biometrical measurements in twin gestation.

(A) Head circumference reference values compared to Chervenak et al.'s curves (in standard deviation 0, -1, -2) [22]; (B) abdominal circumference reference values compared to Hadlock et al.'s curves (in $10^{\text {th }}, 50^{\text {th }}, 90^{\text {th }}$ percentiles) [18].

uncomplicated twin pregnancies were included with limited number of data points, thus a wide variability in the confidence intervals (Figure 4). Their sEFW values were similar to ours up to 32 gestational weeks ( $\pm 100 \mathrm{~g}$ ). However, at 33 weeks, the difference increased with lower values as compared to ours. The second study to include US population [5] applied similar statistical analysis on 1831 twin pregnancies born alive after 28 weeks. Their main limitation was the lack of week-specific data, causing difficulties to compare curves. Recently, third US population normograms were published by Grantz et al. [9]. The authors prospectively evaluated 171 dichorionic twin pregnancies. In this study, a slight deceleration in fetal growth was noted at 32 weeks. Surprisingly, and despite differences in nationality and ethnicity, our reference values were almost identical to Grantz et al.'s curves, with maximal difference measured at 33-36 weeks up to 70 g. Lastly, Gabbay-Benziv et al. [16] published sEFW curves based on a retrospective longitudinal analysis of 5515 twin fetuses. Their values were consistent with ours up to 35 weeks, when large deviation appeared ( $426 \mathrm{~g}$ at 38 weeks).

Two other studies constructed normograms based on Brazilian population. First by Liao et al. [14] was a prospective longitudinal study of 125 uncomplicated twin pregnancies evaluated every 3 weeks from 14 to 38 gestational weeks. Their sEFW values were similar to ours $\pm 50 \mathrm{~g}$ up to 35 gestational weeks. From then on, our values became increasingly higher up to $216 \mathrm{~g}$ difference at 38 gestational weeks. Like fetal weight, FL, also evaluated in the same study, was similar to ours up to 33 weeks of gestation, with increased difference thereafter. The second Brazilian study was a retrospective cross-sectional analysis of 176 dichorionic-diamniotic twin pregnancies (Araujo et al. [10]). Despite the comparison to dichorionic twins only and selection of an ultra-normal cohort (excluding all maternal chronic illness and twin discordance), their results were similar to ours with only minimal differences of $20 \mathrm{~g}$ throughout most gestational weeks. Lastly, Shivkumar et al. [15] conducted a retrospective analysis of 219 twin pregnancies in Canada. Using multilevel linear regression models, their curves yielded increasingly higher values relative to all other curves. This difference may be attributed to selection bias, ethnicity or the study methodology.

To facilitate comparison, we expressed all curves graphically (Figures 3 and 4). Normograms published by Araujo et al. [10], Gabbay-Benziv et al. [16] and the present study have the smallest deviation from Grantz et al. [9], the largest prospective study published on US population (the NICHD fetal growth study).

The considerable variability in SEFW, especially after 32 weeks, may contribute to both false-negative and falsepositive diagnosis of fetal growth restriction, depending on the chosen normograms. For example, a fetal weight of $2100 \mathrm{~g}$ at 34 weeks of gestation implies small for gestational age by some curves (Yarkoni et al. [13], Liao et al. [14], Gabbay-Benziv et al. [16]); however, it fits normal growth for other curves (Grantz et al. [9], Araujo et al. [10], Shivkumar et al. [15]). This inconsistency stresses the urgent need for further research to define normal growth in twin gestations.

Our study also constructed reference values for fetal biometric measurements. These become especially important as many obstetricians use reference values that were constructed based on singleton pregnancies $[18,22]$. It was not surprising that comparison of our curves to Hadlock et al.'s [18] curves for AC yielded small values of AC for every GA in our curves. Likewise, median HC for almost all GA at our study was smaller compared to Chervenak et al.'s [22] curves. This trend was less obvious for the -1 SD and -2 SD, perhaps due to the small cohort evaluated. We also compared our biometrical measurement references to the ones published by Stirrup et al. [7] based on analysis of the Southwest Thames Obstetric Research Collaborative (STORK) multiple pregnancy cohort. This was a retrospective analysis of a large dataset of unselected twin sonographic evaluations conducted in nine medical centers in England. 
Similar values were noted compared to ours for AC, HC and FL (Figure 6).

Our study benefited from the cohort size, the verified GA at evaluation, the standardized sonographic evaluation and the meticulous statistical methodology. The appropriateness of our statistical analysis is based on the verification of a normal distribution after transformation of the weight estimates, calculating the mean fetal weight and the SD for each week of gestation, demonstration of goodness of fit, and presenting the proportion of values that are above or below the fitted percentiles.

Our cohort included an unselected population of women with common co-morbidities (such as hypertension and diabetes). Also, we did not exclude fetuses with growth abnormalities. The use of an unselected population for the construction of growth curves has been a subject for debate among researchers, with both pros and cons expressed. The main advantage of an unselected population is the resemblance to the "normal"

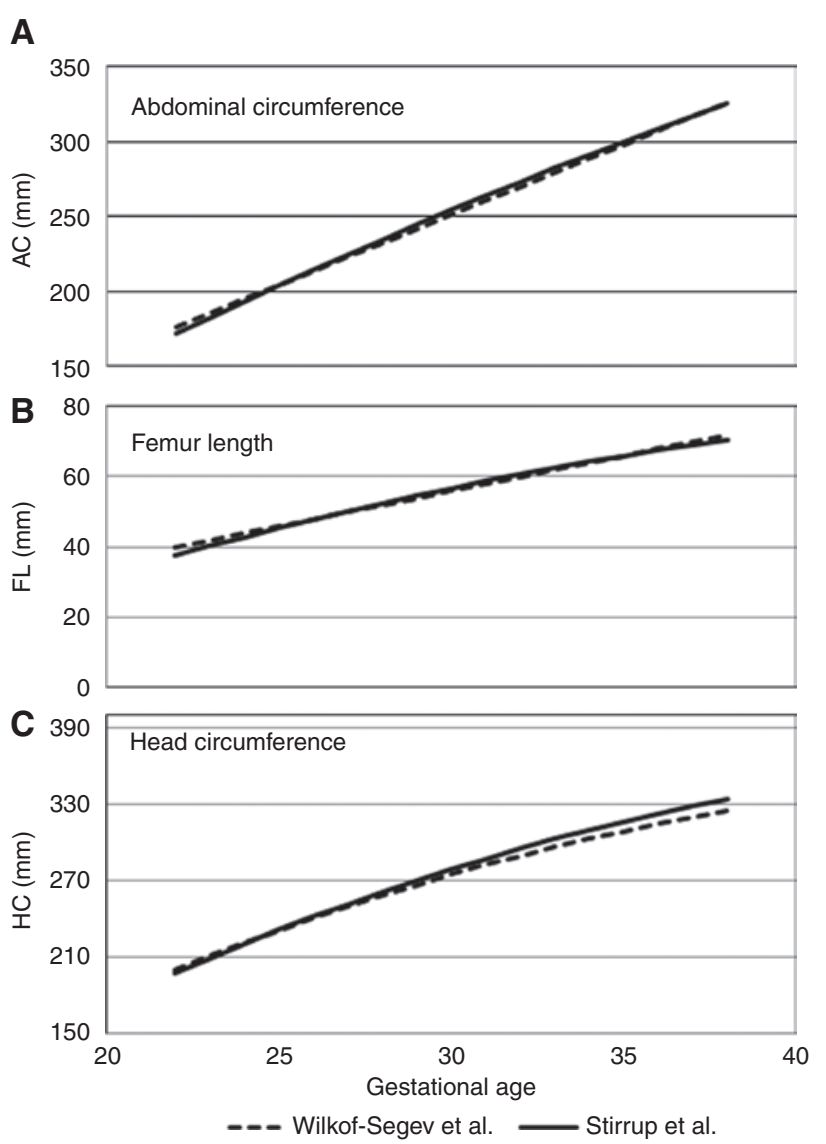

Figure 6: Comparison of reference values of sonographic biometrical measurements in twin gestation.

(A) Abdominal circumference reference values compared to Stirrup et al.'s curves (50 $0^{\text {th }}$ percentile) [7]; (B) femur length reference values compared to Stirrup et al.'s curves ( $50^{\text {th }}$ percentile) [7]; (C) head circumference reference values compared to Stirrup et al.'s curves (50 ${ }^{\text {th }}$ percentile) [7]. population. Exclusion of co-morbidities and pregnancies complicated by fetal growth abnormalities would potentially lead to an "ultra-normal" cohort and construction of growth standards for fetuses under "optimal" conditions. In our study, we aimed to construct reference values for fetal growth that would fit the normal population under normal conditions. Previously, Kiserud et al. discussed this dilemma in their publication of the World Health Organization (WHO) growth charts. Eventually, they decided to include pregnancy complications in their cohort when constructing the WHO growth charts [23]. Despite this and to avoid over-effect of rare exceptional cases, we included only one observation per patient and excluded all outliers that were $>3.5 \mathrm{SD}$ from the mean at each week.

Our study has several limitations. First, our data rely on single hospital population that may have a higher representation of high-risk pregnancies compared to community-followed pregnancies. Data regarding maternal co-morbidities and method of conception might have an influence on in utero growth and fetal weight. However, these data were not available. Also, our study is limited by its retrospective design. As such, we had a fixed number of eligible pregnancies in our cohort with an unequal spread of data across GA. Our data had a larger representation of mid- compared to late-gestation measurements, which may cause bias in SEFW near term with fewer measurements available for analysis and broader confidence intervals. Ideally, we would need a much larger sample size to increase our reference set reliability. Lastly, we did not have enough data on maternal characteristics to construct customized normograms. Despite growing data on their validity, the use of customized curves is still controversial $[24,25]$. Also, we compared our curves to non-customized curves only.

In conclusion, we present new reference values for biometrical measurements and sEFW for twin gestations. Utilizing our curves to clinical practice will translate to clinical decisions on follow-up and timing of delivery, therefore, should be carefully evaluated prior to implementation.

Author contributions: All the authors have accepted responsibility for the entire content of this submitted manuscript and approved submission.

Research funding: None declared.

Employment or leadership: None declared.

Honorarium: None declared.

Competing interests: The funding organization(s) played no role in the study design; in the collection, analysis, and interpretation of data; in the writing of the report; or in the decision to submit the report for publication. 


\section{References}

1. Corsello G, Piro E. The world of twins: an update. J Matern Fetal Neonatal Med 2010;23 (Suppl 3):59-62.

2. The ESHRE Capri Workshop Group. Multiple gestation pregnancy. Hum Reprod 2000;15:1856-64.

3. American College of Obstetricians and Gynecologists. Multiple gestation: complicated twin, triplet, and high-order multifetal pregnancy. ACOG Practice Bulletin No. 56. Obstet Gynecol 2004;104:869-83.

4. American College of Obstetricians and Gynecologists. Perinatal risks associated with assisted reproductive technology. ACOG Committee Opinion No. 324. Obstet Gynecol 2005; 106:1143-6.

5. Min SJ, Luke B, Gillespie B. Birth weight references for twins. Am J Obstet Gynecol 2000;182:1250-7.

6. Walters W. Multiple pregnancy. In: Chamberlain G, editor. Turnbull's obstetrics. Edinburgh: Churchill Livingstone; 1995.

7. Stirrup OT, Khalil A, D’Antonio F, Thailaganathan B. Southwest Thames Obstetric Research Collaborative (STORK). Fetal growth reference ranges in twin pregnancy: analysis of the Southwest Thames Obstetric Research Collaborative (STORK) multiple pregnancy cohort. Ultrasound Obstet Gynecol 201545:301-7.

8. Khalil A, D’Antonio F, Dias T, Cooper D, Thilaganathan B, Southwest Thames Obstetric Research Collaborative (STORK). Ultrasound estimation of birth weight in twin pregnancy: comparison of biometry algorithms in the STORK multiple pregnancy cohort. Ultrasound Obstet Gynecol 2014;44:210-20.

9. Grantz KL, Grewal J, Albert PS, Wapner R, D’Alton ME, Sciscione A, et al. Dichorionic twin trajectories: the NICHD Fetal Growth Studies. Am J Obstet Gynecol 2016;215:221.

10. Araujo Júnior E, Ruano R, Javadian P, Martins WP, Elito Jr J, Pires CR, et al. Reference charts for fetal biometric parameters in twin pregnancies according to chorionicity. Prenat Diagn 2014;34:382-8.

11. American Institute of Ultrasound in Medicine. AIUM practice guideline for the performance of obstetric ultrasound examinations. J Ultrasound Med 2014;32:1083-101.

12. Salomon LJ, Alfirevic Z, Berghella V, Bilardo C, HernandezAndrade E, Johnsen SL, et al. Practice guidelines for perfor- mance of the routine mid-trimester fetal ultrasound scan. Ultrasound Obstet Gynecol 2011;37:116-26.

13. Yarkoni S, Reece E, Holford T, O'Connor T, Hobbins JC. Estimated fetal weight in the evaluation of growth in twin gestations: a prospective longitudinal study. Obstet Gynecol 1987;69:636-9.

14. Liao AW, Brizot Mde L, Kang HJ, Assunção RA, Zugaib M. Longitudinal reference ranges for fetal ultrasound biometry in twin pregnancies. Clinics (Sao Paulo) 2012;67:451-5.

15. Shivkumar S, Himes KP, Hutcheon JA, Platt RW. An ultrasoundbased fetal weight reference for twins. Am J Obstet Gynecol 2015;213:224.

16. Gabbay-Benziv R, Crimmins S, Contag SA. Reference values for sonographically estimated fetal weight in twin gestations stratified by chorionicity: a single center study. J Ultrasound Med 2017;36:793-8.

17. Khalil A, Rodgers M, Baschat A, Bhide A, Gratacos E, Hecher $\mathrm{K}$, et al. ISUOG Practice Guidelines: role of ultrasound in twin pregnancy. Ultrasound Obstet Gynecol 2016;47:247-63.

18. Hadlock F, Harrist R, Sharmann R, Russell LD, Seung KP. Estimation of fetal weight with the use of head, body, and femur measurements - a prospective study. Am J Obstet Gynecol 1985;151:333-7.

19. Altman DG, Chitty LS. Charts for fetal size, 1: methodology. Br J Obstet Gynaecol 1994;101:29-34.

20. Royston P, Wright EM. How to construct 'normal ranges' for fetal variables. Ultrasound Obstet Gynecol 1998;11:30-8.

21. Yeo IK, Johnson R. A new family of power transformations to improve normality or symmetry. Biometrika 2000;87:954-9.

22. Chervenak FA, Jeanty P, Cantraine F, Chitkara U, Venus I, Berkowitz RL, et al. The diagnosis of fetal microcephaly. Am J Obstet Gynecol 1984;149:512-7.

23. Kiserud T, Benachi A, Hecher K, Perez RG, Carvalho J, Piaggio $G$, et al. The World Health Organization fetal growth charts: concept, findings, interpretation, and application. Am J Obstet Gynecol 2018;218:S619-29.

24. Cordiez S, Deruelle P, Drumez E, Bodart S, Subtil D, HoufflinDebarge $\mathrm{V}$, et al. Impact of customized growth curves on screening for small for gestational age twins. Eur J Obstet Gynecol Reprod Biol 2017;215:28-32.

25. Ghi T, Prefumo F, Fichera A, Lanna M, Periti E, Persico N, et al. Development of customized fetal growth charts in twins. Am J Obstet Gynecol 2017;216:514.e1-17. 